\title{
Static and dynamic networks in interbank markets
}

\author{
ETHAN COHEN-COLE \\ Econ One Research, 2040 Bancroft Ave \#200, Berkeley, CA 94708, USA \\ (e-mail:ecohencole@econone.com) \\ ELEONORA PATACCHINI \\ Department of Economics, Cornell University, Ithaca, NY 14853, USA \\ (e-mail:ep454@cornell.edu) \\ YVES ZENOU \\ Department of Economics, Stockholm University and IFN, 10691 Stockholm, Sweden \\ (e-mail:yves.zenou@ne.su.se)
}

\begin{abstract}
This paper proposes a model of network interactions in the interbank market. Our innovation is to model systemic risk in the interbank network as the propagation of incentives or strategic behavior rather than the propagation of losses after default. Transmission in our model is not based on default. Instead, we explain bank profitability based on competition incentives and the outcome of a strategic game. As competitors' lending decisions change, banks adjust their own decisions as a result: generating a "transmission" of shocks through the system. We provide a unique equilibrium characterization of a static model, and embed this model into a full dynamic model of network formation. We also determine the key bank, which is the bank that is crucial for the stability of the financial network.
\end{abstract}

Keywords: financial networks, interbank lending, interconnections, network centrality, key banks

JEL Classification: G10, C21, D85.

\section{Introduction}

Since the onset of the financial crisis in 2007, the discourse regarding bank safety has shifted strongly from the riskiness of financial institutions as individual firms to concerns about systemic risk. ${ }^{1}$ As the crisis evolved, the public debate did as well, with concerns about systemic risk evolving from too-big-to-fail (TBTF) considerations to too-interconnected-to-fail (TITF) ones. The academic and policy literature has followed, with the growth of papers that discuss network linkages in the

1 Indeed, there is a wide range of research on the importance of interbank markets, including some that address the systemic risk inherent to these markets. Some examples include Allen \& Gale (2000), Freixas et al. (2000), Iori \& Jafarey (2001), Boss et al. (2004), Furfine (2003), Iori et al. (2006), Soramäki et al. (2007), Pröpper et al. (2008), Cocco et al. (2009), Mistrulli (2011), and Craig \& Von Peter (2010). Each of these discuss some network properties or discuss the importance of these markets to systemic risk evaluation. 
financial system. ${ }^{2}$ This literature's largest success has been identifying and describing the properties of financial networks. Prior to the crisis, most observers knew that financial institutions were connected, and that knowledge has now expanded to recognize, for example, that the banking system is largely tiered, that the Credit Default Swap (CDS) market exhibits small-world properties (Peltonen et al., 2013), and that the largest 50 or so institutions in the EU are highly connected (Alves et al., 2013). A variety of other papers find similar features in other markets and sub-components of the same markets (see Alves et al. (2013) for a good review of the recent literature).

A second success of the recent literature is a schematic linking of network properties and patterns to economic outcomes. By successfully appropriating the terminology and methods of other fields and applying them to the graph of various financial markets, we are beginning to learn the broad correlations between networks and outcomes. For example, one can look at the structure of the network before, during and after the crisis and then correlate the observed economic outcomes with the network structure. See for example Puhr et al. (2012) and Fricke \& Lux (2012). The former shows changes through the crisis and the latter through stability, though they treat different markets.

Finally, a third branch of research has undertaken the difficult task of modeling the economics that drive agent participation in the network. The current financial networks literature is largely based on random network and preferential attachment models which both use models from the applied mathematics and physics literature (Albert \& Barabási, 2002; Easley \& Kleinberg, 2010; Newman, 2010). The preferential attachment model (Barabási \& Albert, 1999) is effectively based on a random network approach, since agents form links in a probabilistic way leading to more popular nodes being more likely to be chosen (the so-called rich-get-richer model). To this class of models belong simplified networks with simple shocks, including models of cascading default.

This paper has two goals. First, we seek to add to a portion of the field that has received little attention - the link between propagation of financial risk and agent incentives on a network. It is well known and accepted that banks act strategically given the market and regulatory incentives they face. We apply the new methods of optimization in networks (Goyal, 2007; Jackson, 2008; Jackson \& Zenou, 2015) to the interbank market with a point-in-time spatial model of homogeneous banks and no defaults. Using these methods, we are able to precisely identify the equilibrium quantity of lending attributable to the network structure.

The closest papers to our in the literature are Acemoglu et al. (2015) and Elliott et al. (2014), as well as an earlier paper by Gai \& Kapadia (2010). These papers explore the propagation of shocks in financial institutions networks. Networks in these papers are debt holdings or interbank lending, and shock propagation originates from an institution's failure to pay some or all of its debts. The three

2 A general perception and intuition has emerged that the interconnectedness of financial institutions is potentially as crucial as their size. A small subset of recent papers that emphasize such interconnectedness include Acemoglu et al. (2015), Adrian \& Brunnermeier (2009), Allen et al. (2012), Amini et al. (2012), Boyson et al. (2010), Cabrales et al. (2013), Cohen-Cole et al. (2014), Danielsson et al. (2009), and Elliott et al. (2014). 
papers' principal results reflect a spectrum of potential shocks from small to huge. Acemoglu et al. (2015) focus on the extremes and Elliott et al. (2014) highlight that intermediate cases can be particularly worrisome. These papers also discuss the gamut of network structures in terms of the consequences of shocks. Gai \& Kapadia (2010) highlight that the location of a shock and connectivity of a network will impact the outcome of a shock. Elliott et al. (2014) add a dimension to the analysis by including the level of cross-holdings of the network in addition to diversification of holdings. ${ }^{3}$

We complement this line of research by exploring a different dimension of shocksones that do not emerge from a bankruptcy or other failure to pay. Instead, we explore shocks that occur as a result of financial incentives in the absence of default. Financial institutions regularly adjust market participation. Indeed, markets are characterized by increasingly fast re-optimizations of portfolios and asset holdings. These changes have lightning-fast impacts; as a result, changes in bank incentives can lead to changes in holdings long before any defaults take place or even in the complete absence of defaults. Bank runs and repo runs are simple examples of the potential adverse consequences of these actions.

By describing the bank optimization without default, we can then determine the impact on the network structure itself. In addition to being able to comment on the extant properties of different network structures, the model here can link the network structure to specific bank incentives and behaviors. For policy makers, this linkage is crucial. Much as competition regulators need an understanding of the behavior of market participants in order to make informed policy decisions as to the regulation of a given industry, a bank regulator must have information on bank behavior and incentives in order to make policy decisions. Our paper is among a relatively small set of papers to both explicitly model a set of bank behaviors and describe how behaviors change as a function of a complex market structure.

Our second goal is to use the description of bank behavior within a network to illustrate, how an understanding of this behavior can be formalized into a measure of systemic risk. In particular, we provide a measure that formally links the structure of a network to the propagation of incentives. We again look at a system without defaults, and illustrate how small changes in uncertainty, risk, or behavior can propagate through a network; this propagation leads to changes in volumes and prices in concrete and measurable ways. Indeed, we provide a closed form solution for each. This propagation is well understood from an institutional perspective; what remains is to link this type of phenomenon explicitly to network theoretical tools so that these phenomena can be understood structurally. Institutionally, there is a broad acceptance of the presence of contagion effects; fear of risk can spread rapidly in a variety of forms. The structural view is important because the exact topology of the network can fundamentally alter incentives and prices. Much in the same way that Elliott et al. (2014) calculate how particular network structures can be linked to particular default cascades, we provide a mechanism to link a shock without default

3 While we do not explore in this paper, our paper shares with Elliot et al. (2014) the possibility of non-monotonicity of impact from shocks. They occur in our model due to the generalizability of network structure and in Elliot et al. (2014) because of the presence of two dimensions of network connectivity. 


\begin{tabular}{|c|c|}
\hline \multicolumn{1}{|c|}{ Assets } & Liabilities \\
\hline Cash & Deposits \\
\hline Loans & Interbank borrowing \\
\hline \multirow{2}{*}{ Interbank loans $=q_{i}$} & \\
& Equity $=e_{i}$ \\
\hline
\end{tabular}

Fig. 1. Balance sheet.

to particular cascades. This provides a measure of total systemic risk, as well as illustrates a method to calculate the contribution of individual banks to this total. Importantly, both of these measures emerge directly from the optimization problem of banks.

To further capture the fact that financial networks are rapidly changing, we take the modeling exercise another step forward. As shocks hit a system, the existing pattern of network links will evolve over time. As such, reduced form and/or static spatial models of systemic risk may be insufficient for understanding the importance of interconnectedness on financial markets. With this in mind, we explicitly embed our static model into a complete dynamic model of network formation. Thus, we are able to characterize not only the equilibrium pattern of behavior at each point in time, but also describe how this behavior evolves over time. As banks form and break links, the structure of the network will change, and the nature of systemic risk with it. Our model is useful in that we can discuss how systemic events emerge even in the absence of defaults (e.g. runs on the bank, flights to quality, etc.).

Once we have developed the static and dynamic models, we look at the impact of the removal of a bank from the network on total activity. We derive an exact formula for the "key bank", i.e. the bank which once removed from the network reduces total activity (here total volume of loans) the most. This policy can help a regulatory authority (such as the European Central Bank (ECB)) to decide which bank should be bailed out in case of a financial crisis.

\section{Static model}

\subsection{Notation and model}

We begin with a simple static model of a bank whose balance sheet is given in Figure 1. On the asset side of the balance sheet, we include cash, loans and interbank loans. On the liability side, deposits, interbank borrowing and equity.

Our primary object of interest will be either interbank loans or interbank deposits. In addition, we specify a basic leverage constraint for each bank as:

$$
\xi e_{i} \geqslant \operatorname{assets}
$$

where $e_{i}$ is the equity of bank $i$ and $\xi$ is the leverage constraint. For simplicity, we group cash and loans into a single variable $X_{i}$ (i.e. loans + cash $=X_{i}$ ). Then we can write that interbank loans at each point in time must satisfy two criterion. One, 
given a value for liabilities and for $X_{i}$,

$$
q_{i}=\text { liabilities }_{i}-X_{i}
$$

The equality condition simply means that banks must match assets and liabilities. The assumption that interbank loans are the remaining choice on the balance sheet reflects the nature of this market. Precise deposits balances are determined by customer preferences, non-interbank loans are typically much longer maturity and cannot be underwritten or sold on a moment's notice with any reliability, and equity takes weeks or months to issue. Thus, in the perspective of a day or two, one of the only free variables for a bank to clear its balance sheet is the interbank market. ${ }^{4}$

Two, the leverage constraint requires that

$$
\xi e_{i} \geqslant X_{i}+q_{i}
$$

This reflects the fact that banks cannot lend funds greater than some multiple of their equity. For example, in the 2000s, European banks were not bound explicitly by a leverage constraint. However, Basel capital constraints formed a type of upper bound on the quantity of lending possible. We include this feature particularly because new Basel III regulations explicitly discuss additional capital requirements for systemically important financial institutions (SIFIs). The borrowing side obviously has no such constraint.

As we develop the model, two key features will emerge: global strategic substitutability and local strategic complementarities. These will show that, as total quantities in the market increase, prices will fall. However, at the local level, between two agents, there will be an incentive to increase prices when quantities increase due to the complementarity effect. The model will find an equilibrium where these effects are balanced.

To our knowledge, the fact that we incorporate both local and global components adds to the financial networks literature; by incorporating both the direct network influences as well as the system-wide effects, our model is particularly suited to the description of financial markets. These markets are influenced both by prices (global) and network impacts (local).

We look at a population of banks. We define for this population a network $g \in \mathscr{G}$ as a set of ex-ante identical banks $N=\{1, \ldots, n\}$ and a set of links between them. We assume at all times that there are least two banks, $n \geqslant 2$. Links in this context can be defined in a variety of ways. In other work, they have represented the exchange of a futures contract (Cohen-Cole et al., 2014). In the banking networks that we study, the links will represent the presence of a interbank loan. This means, in particular, that the network is directed because bank $i$ may make a loan to bank $j$ (there is thus a link between them) while bank $j$ may not make a loan to bank $i$.

In the language of graph theory, in a directed graph, a link has two distinct ends: a head (the end with an arrow) and a tail. Each end is counted separately. The sum of head endpoints count toward the indegree and the sum of tail endpoints count toward the outdegree. Because here we consider loans between banks, we will only

4 We abstract for now from the ability to borrow from the central bank. This is an alternate mechanism to match the balance sheet. However, this type of borrowing typically comes at a penalty rate. We return to penalty rate borrowing in the policy section at end. 
consider outdegrees. Formally, we denote a link from bank $i$ to bank $j$ as $g_{i j}=1$ if bank $i$ has given a loan to bank $j$, and $g_{i j}=0$, otherwise. The set of bank $i$ 's direct links is: $N_{i}(g)=\left\{j \neq i \mid g_{i j}=1\right\}$. The cardinality of this set is denoted by $n_{i}(g)=\left|N_{i}(g)\right|$. In other words, the outdegree of bank $i$, denoted by $n_{i}(g)$, is the number of loans bank $i$ has given, that is $n_{i}(g)=\sum_{j} g_{j i}$. The $n$-square adjacency matrix $\mathbf{G}$ of a network $g$ keeps track of the direct connections in this network. Because the network is directed, $\mathbf{G}$ is asymmetric. ${ }^{5}$

We hypothesize that these direct links produce a reduction in costs of the collaborating banks. In other words, we assume that a financial institution's cost of doing business is lowered through their financial connections to other institutions, and by their neighbors doing more business. Although the following is outside the model, it is intuitive that if a financial institution $i$ has a neighbor that does a lot of business, that institution will be able to lend money more reliably to $i$ when $i$ requires it, reducing the cost to $i$ of using the interbank market to make up balance sheet shortfalls. This creates a new mechanism through which shocks can be transmitted. We believe that this mechanism is particularly appealing because it provides a natural motivation for financial connections to be established and can also help explain the observed structure of the interbank market.

This assumption seems to be strongly supported empirically. Treasury operations for a small deposit-taking entity with a single link typically consist of a daily phone call from the bank CFO to a regular, and typically much larger, counterparty to arrange to lending to the larger counterparty. For example, in the case of e-MID, ${ }^{6}$ the small bank must incur the fixed costs of participating even though it has only a single link. These operations are expensive to maintain as it takes the direct participation of senior management of the small bank to generate the loan. As banks increase their connections; however, the average unit costs associated with managing a treasury operation strongly decrease in the number of counterparties. Some evidence for this is seen in the fact that only a few banks serve as de-facto moneycenter banks. These institutions are capable of managing a multiplicity of links because of the high fixed and low average cost per counterparty.

We will model the quantity choice (i.e. volume of loans) based on competition in quantities of lending a la Cournot between $n$ banks with a single homogenous product (a loan). We do not model the borrowing market but our analysis would be the same if we only focused on borrowers and not lenders. The distinction between lenders and borrowers is useful for three reasons. First, it allows us to look separately at what happens to each side of the market. Second, it allows us to use well-established competition frameworks, such as Cournot, which are based on the idea of a group of firms competing for customer business. Looking only at one side of the market (here interbank loans) allows this view. Third, looking at each side of the market individually reflects the fact that we need gross lending amounts to understand competitive forces. If a bank borrows $\$ 100$ and lends $\$ 99$,

5 Vectors and matrices will be denoted in bold and scalars in normal text.

6 The e-MID SPA (or e-MID) was the reference marketplace for liquidity trading in the Euro area during the period 2002-2009. It was the first electronic marketplace for interbank deposits (loans), a market that has traditionally been conducted bilaterally. 
to understand the network, we need to know both quantities; a netted $\$ 1$ borrowing does not capture the complexity and scale of the interactions.

We assume the following standard linear inverse market demand where the market price is given by:

$$
p=\theta-\sum_{j \in N} q_{j}
$$

where $\theta>0$. This means that we assume that the loan market is integrated since there is a single market-clearing price and each bank is path-connected with any other bank (network component). This is true in the interbank market, for example the eMID market. As stated above, we only look at one side of the market. If we analyze the lenders' behavior only, then $p$ determines the price (interest rate) of loans between banks. If we study the borrowers' behavior, then $p$ reflects the price of borrowing between banks. Observe that issues like the riskiness of the lenders or borrowers are ignored here. As stated above, in this paper, we focus on the lenders' behavior.

The marginal cost of each bank $i \in N$ is $c_{i}(g)$. The profit function of each bank $i$ in a network $g$ is therefore given by:

$$
\begin{aligned}
\pi_{i}(g) & =p q_{i}-c_{i}(g) q_{i} \\
& =\theta q_{i}-\sum_{j \in N} q_{i} q_{j}-c_{i}(g) q_{i}
\end{aligned}
$$

where $q_{i}$ is the loan quantity produced by bank $i$. We assume throughout that $\theta$ is large enough so that price and quantities are always strictly positive.

Our specification of inter-related cost functions is as follows. The cost function is assumed to be equal to:

$$
c_{i}(g)=c_{0}-\phi\left[\sum_{j=1}^{n} g_{i j} q_{j}\right]
$$

where $c_{0}>0$ represents a bank's marginal cost when it has no links while $\phi>0$ is the cost reduction induced by each link formed by a bank. The parameter $\phi$ could be bank specific as well so that $\phi_{i}$, but for simplicity of notation, we do not report this case.

Equation (3) means that the marginal cost of each bank $i$ is a decreasing function of the quantities produced by all banks $j \neq i$ that have a direct link with bank $i$. As stated above, this is because the operational costs of a trading floor or treasury operation decline per dollar of loan as loan size increases. This is the specification that drives the functional relationships between banks.

To ensure that we obtain a reasonable solution, we assume that $c_{0}$ is large enough so that $c_{i}(g) \geqslant 0, \forall i \in N, \forall g \in \mathscr{G}$. The profit function of each bank $i$ can thus be written as:

$$
\begin{aligned}
\pi_{i}(g) & =p q_{i}-c_{i}(g) q_{i} \\
& =\theta q_{i}-\sum_{j \in N} q_{i} q_{j}-c_{0} q_{i}+\phi \sum_{j=1}^{n} g_{i j} q_{i} q_{j} \\
& =a q_{i}-q_{i}^{2}-\sum_{j \neq i} q_{i} q_{j}+\phi \sum_{j=1}^{n} g_{i j} q_{i} q_{j}
\end{aligned}
$$

where $a \equiv \theta-c_{0}>0$. 
We highlight a few features of Equation (4). First, we can see that profits are a negative function of total loans. This we call global strategic substitutability, as the effect operates only through the market and not through the direct links that form the network. So as $q_{j}$ increases, $\frac{\partial \pi_{i}(g)}{\partial q_{i}}$ is reduced as demand falls.

Second, we can see that profit is increasing in the quantity of direct links, via the cost function impact. This we refer to as local strategic complementarities since if $j$ is linked with $i$, then if $q_{j}$ increases $\frac{\partial \pi_{i}(g)}{\partial q_{i}}$ is increased because of the reduction in the cost. Total profits are of course, dependent on the two jointly.

Third, we can define $\sigma_{i j}$ as the cross partial of profitability with respect to a bank's quantity change and another bank's quantity change. We have:

$$
\sigma_{i j}=\frac{\partial^{2} \pi_{i}(g)}{\partial q_{i} \partial q_{j}}=\left\{\begin{array}{ccc}
\bar{\sigma}=-1+\phi & \text { if } & g_{i j}=1 \\
\underline{\sigma}=-1 & \text { if } & g_{i j}=0
\end{array}\right.
$$

so that $\sigma_{i j} \in\{\underline{\sigma}, \bar{\sigma}\}$, for all $i \neq j$ with $\underline{\sigma} \leqslant 0$.

This last feature highlights the mechanism of the model. A shock to a connected bank changes the incentives of a bank to lend, precisely through the function (5). Notice that the model generates systemic risk insofar as shocks that impact a given bank, such as an exogenous decrease in capital and ability to lend, pass through to the rest of the market through a competition mechanism. The global effect of the reduction in lending by a single bank is an increase by others. The local effect, however, that passes through the network linkages, is that costs increase. As a result, loan volumes of direct network links decline as well. Once network links change their choices, their links do so as well, and so on.

This dual effects and implicit tradeoffs have policy implications. Competition in a network context/connected industries requires an understanding of how firms' interconnections impact their pricing and volume decisions. These linkages can lead to local cascades and reductions in total lending even when the system as a whole is not at risk.

\subsection{Equilibrium loans}

Consider a Cournot game in which banks choose a volume of interbank lending conditional on the actions of other banks. This game requires common knowledge of the actions of other banks. Agents have the defined profit function in Equation (4), which implies that cost is intermediated by the network structure.

It is easily checked that the first-order condition for each bank $i$ is given by:

$$
q_{i}^{*}=a-\sum_{j=1}^{j=n} q_{j}+\phi \sum_{j=1}^{n} g_{i j} q_{j}
$$

We now characterize the Nash equilibrium of the game. Denote by $\mathbf{b}(g, \phi)$ the $(n \times 1)$ vector of Katz-Bonacich centralities, which is formally defined in Appendix A. For all $\mathbf{b}(g, \phi) \in \mathbb{R}^{n}$, denote $b(g, \phi)=b_{1}(g, \phi)+\cdots+b_{n}(g, \phi)$ as the sum of its coordinates.

\section{Proposition 1}

Consider a network game where links are represented by loans between $n$ banks and where the profit function of each bank $i$ is given by Equation (4). Then this 
game has a unique Nash equilibrium in pure strategies if and only if $\phi \omega(\mathbf{G})<1$. This equilibrium $\mathbf{q}^{*}$ is interior and given by:

$$
\mathbf{q}^{*}=\frac{a}{1+b(g, \phi)} \mathbf{b}(g, \phi)
$$

The equilibrium profit is then given by:

$$
\pi_{i}^{*}=\left(q_{i}^{*}\right)^{2}=\frac{a^{2} b_{i}^{2}(g, \phi)}{[1+b(g, \phi)]^{2}}
$$

This result is a direct application of Theorem 1 in Ballester et al. (2006). Appendix $\mathrm{B}$ shows in more detail how the first order condition can be written as a function of Katz-Bonacich centrality. It also provides an example.

This solution is useful for a couple of reasons: one, notice that this equation provides a closed form solution to the game with any number of banks and to calculate output, only the matrix of interconnections $\mathbf{G}$ and the bank specific cost functions are needed. Two, this equation provides the basis for estimation of any network linked bank decision. We explore this implication in more detail below in Section 3.

A key parameter in this equilibrium result is $\phi$, the coefficient in the Equation (18) that measures how much of a shock to a given bank is passed on to connected banks. While we don't explore in this paper, our paper shares with Elliot et al. (2014) the possibility of non-monotonicity of impact from shocks. They occur in our model due to the generalizability of network structure and in Elliot et al. (2014) because of the presence of two dimensions of network connectivity. The equilibrium in our model is a measure of the pass-through rate, but notice that diverse network structures lead to potentially widely diverse impacts of shocks that depend on the location and severity of the shock as well as the structure of the network.

To illustrate this issue, denote by $q^{N O *}$ the Nash equilibrium in loan quantities where there is no network and by $q_{i}^{N E T *}$ the same quantity where banks are connected by a network (defined by Equation (7)). We have the following result, which proof is given in Appendix C.

Proposition 2

Assume that $\phi \omega(\mathbf{G})<1$. Then,

$$
q_{i}^{N E T^{*}}=q_{i}^{N O *}+\frac{\phi}{(1+n)}\left[n \sum_{j=1}^{n} g_{i j} q_{j}^{*}-\sum_{k \neq i}^{n} \sum_{j=1}^{n} g_{k j} q_{j}^{*}\right]
$$

In the case of a dyad, i.e. we have:

$$
q^{N E T *}=\frac{3}{(3-\phi)} q^{N O *}
$$

Proposition 2 illustrates that $\phi$ is a multiplier and, in our context, is a measure of systemic risk that propagates risk through incentives. The intuition of Equation (9) is clear. Total output is higher with networks than without networks and the difference is measured as $q^{N E T^{*}}-q^{N O^{*}}=\left(\frac{\phi}{1+n}\right) \sum_{i=1}^{n} \sum_{j=1}^{n} g_{i j} q_{j}^{*}>0$. In other words, total lending increases by this value when network effects are present. This 
implies that prices of loans are much lower with networks since $p^{N O^{*}}=p^{N E T *}+$ $\left(\frac{\phi}{1+n}\right) \sum_{i=1}^{n} \sum_{j=1}^{n} g_{i j} q_{j}^{*}$, which creates even more interactions (i.e. loans). As a result, profits are also higher with networks. The implications are clear. In normal times, the system relies on network structure to boost lending and profits - without the network, both would be lower. As a result, even in the absence of any actual losses, shocks to structure are costly in part because they unwind this benefit at a rapid pace. We continue this notion by articulating a measure of system risk that measures the system's fragility vis-a-vis the collapse or contraction of the network benefit.

In Equation (10), we consider the dyad, i.e. the case of two banks $A$ and $B(n=2)$. In that case, the multiplier is equal to $3 /(3-\phi)>1$. One can see that this multiplier increases in $\phi$ so that the higher is $\phi$, the higher is the quantity of loans that will be given to each bank. This means, in particular, that if there is a shock to this economy, $\phi$, the systemic risk, will propagate the risk at a factor of $3 /(3-\phi)$. In other words, if for example $\phi=0.5$, then an isolated bank will provide a volume of loans equal to $q^{N O *}$ while if it has only a single connection, its volume increases by $20 \%$.

\subsection{Equilibrium prices}

One of the powerful features of the model is that it provides a structural link between the network pattern and the equilibrium market price for interbank loans. Changing the network structure changes equilibrium prices. Indeed, this is true because these prices incorporate the network benefit. Throughout this paper, our cost function is closely related to our findings. We emphasize this tight relationship between a cost function and the incentives to lend in a financial network not because this is the sole possible description of the market. Instead, it enables us to illustrate the fact that incentives impact prices/volumes through network architecture in a clear and measurable way. This can be observed through two features of the model. First, the equilibrium quantity for each bank is expressed precisely in Equation (7). As the sum of these quantities change, the global effect, as in any market, will be to influence prices. This is what we labeled global strategic substitutability, above. Two, the individual patterns of links in the network will influence the local loan decisions. These local strategic complementarities also influence aggregate prices.

To be more precise, using the linear inverse market demand of Equations (2) and (7), we obtain the following equilibrium price of loan transactions:

$$
p^{*}=\theta-\sum_{j \in N} q_{j}^{*}=\theta-\frac{a b(g, \phi)}{1+b(g, \phi)}
$$

where $a=\left(\theta-c_{0}\right)$.

\section{Example 1}

Consider the two following directed networks: 


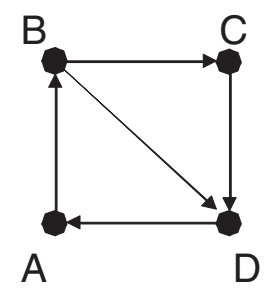

Quasi-Circle

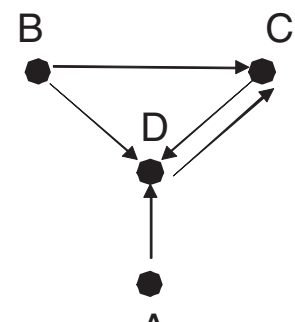

A

Quasi-Star

Fig. 2. Two different directed networks.

The network on the left panel is a quasi-circle (and its adjacency matrix is denoted by $\mathbf{G}^{C}$ ) while the network on the right panel is a quasi-star (and its adjacency matrix is denoted by $\mathbf{G}^{S}$ ). We have: ${ }^{7}$

$$
\mathbf{G}^{C}=\left[\begin{array}{cccc}
0 & 1 & 0 & 0 \\
0 & 0 & 1 & 1 \\
0 & 0 & 0 & 1 \\
1 & 0 & 0 & 0
\end{array}\right] \quad \text { and } \quad \mathbf{G}^{S}=\left[\begin{array}{cccc}
0 & 0 & 0 & 1 \\
0 & 0 & 1 & 1 \\
0 & 0 & 0 & 1 \\
0 & 0 & 1 & 0
\end{array}\right]
$$

where the first row corresponds to bank $A$, the second row to bank $B$, etc. Observe that we have a directed network (since loans are by definition directed) and thus the adjacency matrices are asymmetric. We focus on outdegrees only, i.e. links (i.e. loans) that go from one bank to the other one. In other words, we analyze the lending market. For example, in the quasi-circle network, the first row of $\mathbf{G}^{C}$ indicates that bank $A$ has given a loan to bank $B$ only while, the second row of $\mathbf{G}^{C}$ shows that bank $B$ has given a loan to banks $C$ and $D$. If, for example, we calculate $\mathbf{G}^{C} \mathbf{1}$, where $\mathbf{1}$ is a vector of 1 , then each row gives the total number of loans each bank has given to other banks in network $\mathbf{G}^{C}$. The same interpretation can be made for $\mathbf{G}^{S}$. Observe also the two networks have the same total number of banks (4) and have the same total numbers of loans (5) but have a different structure.

In this framework, the Katz-Bonacich centrality is a measure of "popularity" since the most central bank (i.e. node) is the one that makes the higher number of loans (i.e. links). Fortunately, the symmetry of the adjacency matrix does not play any role in the proof of Proposition 1 and thus the results are true for both directed and undirected networks. We can see here how prices vary as a function of even relatively small changes in network structure. Using Proposition 1, the unique Nash equilibrium is given by:

$$
\left[\begin{array}{c}
q_{A}^{C *} \\
q_{B}^{C *} \\
q_{C}^{C *} \\
q_{D}^{C *}
\end{array}\right]=\frac{\left(\theta-c_{0}\right)}{5+5 \phi+6 \phi^{2}+2 \phi^{3}-\phi^{4}}\left[\begin{array}{c}
1+\phi+2 \phi^{2}+\phi^{3} \\
1+2 \phi+2 \phi^{2}+\phi^{3} \\
1+\phi+\phi^{2} \\
1+\phi+\phi^{2}+\phi^{3}
\end{array}\right]
$$

7 The largest (noncomplex) eigenvalue for $\mathbf{G}^{C}$ is 1 and for $\mathbf{G}^{S}$, it is also 1 . As a result, the eigenvalue condition $\phi \omega(\mathbf{G})<1$ is $\phi<1$ for both networks. 
for the quasi-circle network and

$$
\left[\begin{array}{c}
q_{A}^{S *} \\
q_{B}^{S *} \\
q_{C}^{S *} \\
q_{D}^{S *}
\end{array}\right]=\frac{\left(\theta-c_{0}\right)}{5}\left[\begin{array}{c}
1 \\
1+\phi \\
1 \\
1
\end{array}\right]
$$

for the (quasi)star-shaped network. The quasi-circle network where bank $B$ has the highest Katz-Bonacich centrality (and thus provides the highest number of loans) is less symmetric than the (quasi)star-shaped one. Bank $A$ is the second most active bank because it provides a loan to $B$ - followed by bank $D$ and then bank $C$. On the contrary, for the quasi-star network, banks $A, C$, and $D$ provide the same number of loans while bank $B$ has the highest Katz-Bonacich centrality. This is because they all lend to the same bank, $D$.

What is interesting here is the impact of network structure on the aggregate equilibrium price of loans. In the circle network, each loan is priced at

$$
p^{C^{*}}=\frac{\left(1-\phi^{3}-\phi^{4}\right) \theta+\left(4+5 \phi+6 \phi^{2}+3 \phi^{3}\right) c_{0}}{5+5 \phi+6 \phi^{2}+2 \phi^{3}-\phi^{4}}
$$

where $\theta$ is the market demand from Equation (2) and $\phi$ is the coefficient in the Equation (18). For the star-shaped network, we obtain:

$$
p^{S *}=\frac{(1-\phi) \theta+(4+\phi) c_{0}}{5}
$$

As a result, with four banks $A, B, C$, and $D$, depending on the network structure, the price for loans can differ. Indeed, it is easily verified that $p^{S^{*}}>p^{C^{*}}$. This reflects the fact that the quasi-star network induces less competition and thus less loan output than the quasi-circle network. This example also highlights that cost complementarities are the key force behind the impact of networks on competition.

\subsection{Equilibrium behavior with leverage constraints}

Remember that we have a leverage constraint given by Equation (1). We need to check that the Nash equilibrium satisfies this condition. Define $\bar{q}_{i} \equiv \xi e_{i}-X_{i}$. Since $\xi, e_{i}$ and $X_{i}$ are purely exogenous variables, we consider three possibilities.

\section{Proposition 3}

Assume that $\phi \omega(\mathbf{G})<1$. Then,

i. If no banks are constrained by the leverage constraint, i.e. the equilibrium quantity of loans $q_{i}^{*}$, defined by Equation (7), is such that $q_{i}^{*} \leqslant \bar{q}_{i}$, for all $i=1, \ldots, n$, then all banks play the Nash equilibrium quantity of loans $q_{i}^{*}$ defined by Equation (7).

ii. If all banks are constrained so that $q_{i}^{*}>\bar{q}_{i}$, for all $i=1, \ldots, n$, in equilibrium, all banks provide loans equal to $q_{i}^{*}=\bar{q}_{i} \equiv \xi e_{i}-X_{i}$.

iii. If some banks are constrained by the leverage constraint and some are not, then banks for which $q_{i}^{*} \leqslant \bar{q}_{i}$ will provide loans made by $q_{i}^{*}$, defined in Equation (7), while those for which $q_{i}^{*}>\bar{q}_{i}$, will provide $q_{i}^{*}=\bar{q}_{i} \equiv \xi e_{i}-X_{i}$. 
This difference between constrained and unconstrained suggests that increasing the fraction of constrained banks leads to a smaller fraction of banks propagating incentives through the network. This can have a range of potential impacts on total systemic risk and the allocation of risk in the system. Indeed, when $\xi$ increases, more and more banks are constrained in their loan possibilities and are more likely to hit the leverage constraint so that $q_{i}^{*}=\bar{q}_{i} \equiv \xi e_{i}-X_{i}$. Interestingly, this depends on the network structure so that the same bank with the same leverage constraint can behave differently depending on the network it belongs to. Consider again Example 1 (Figure 2) and assume for bank $C$ that ${ }^{8}$

$$
\frac{\left(\theta-c_{0}\right)\left(1+\phi+\phi^{2}\right)}{5+5 \phi+6 \phi^{2}+2 \phi^{3}-\phi^{4}}<\bar{q}_{C}<\frac{\left(\theta-c_{0}\right)}{5}
$$

This implies that $q_{C}^{S *}>\bar{q}_{C}$ and $q_{C}^{C *}<\bar{q}_{C}$ and thus, in equilibrium,

$$
q_{C}^{C *}=\frac{\left(\theta-c_{0}\right)\left(1+\phi+\phi^{2}\right)}{5+5 \phi+6 \phi^{2}+2 \phi^{3}-\phi^{4}} \text { and } q_{C}^{S *}=\bar{q}_{C} \equiv \xi e_{C}-X_{C}
$$

In other words, the same bank $C$ in the quasi-circle network will provide its Nash equilibrium quantity of loans, but in the star-shaped network will hit the leverage constraint and will lend loans so that $q_{C}^{S *}=\bar{q}_{C}$. This is true for a given $\xi$. When $\xi$ increases then banks are more likely to hit the leverage constraint and will not provide their "optimal" (i.e. Nash equilibrium) quantity of loans. The policy implication is that leverage constraints limit lending not only via directly constrained banks by also by an additional amount as these restrictions propagate through the network.

\section{Framework for empirical analysis}

We begin by defining a network of banks. Banks conduct transactions with other banks nearly continuously; as such, we make an assumption about what defines a network. Since the vast majority of transactions are overnight transactions and banks use the interbank loan market for rectifying deposit imbalances, one can summerize that a reasonable network is characterized by the transactions that occur in a short time frame. A one-day time period is a natural time period to start with. That said, many overnight interbank loans are rolled over the following day. While the lending bank typically has the option to withdraw funding, the persistence in relationships implies that the networks that determine lending choices may be slightly longer than a day. We will use one day as a benchmark measure of networks.

Further supporting the use of a short-term network measure, the model above takes the interbank lending/borrowing decision as the tool to balance the bank's assets and liabilities, taking the remainder of the balance sheet as given. Once we consider other assets and liabilities with longer maturities, alternate network measures may be important.

8 Observe that, since $\phi<1$, we have:

$$
\frac{\left(\theta-c_{0}\right)\left(1+\phi+\phi^{2}\right)}{5+5 \phi+6 \phi^{2}+2 \phi^{3}-\phi^{4}}<\frac{\left(\theta-c_{0}\right)}{5}
$$


Assume that there are $K$ networks in the economy, defined by the number of days. Each network contains $n_{k}$ banks. We can then estimate the direct empirical counterpart of the first-order condition in the static model above, Equation (6):

$$
q_{i, \kappa}=c+\phi \frac{1}{g_{i, k}} \sum_{j=1}^{n_{\kappa}} g_{i j, \kappa} q_{j, \kappa}+v_{i, \kappa}, \quad \text { for } i=1, \ldots, n_{\kappa} ; \kappa=1, \ldots, K
$$

where $c=\frac{1}{2} a-\frac{1}{2} \sum_{j=1, j \neq i}^{n} q_{j}$. This equation indicates that the equilibrium quantity choice of a bank is a function of quantity choices of others in the same market. We denote as $q_{i, \kappa}$ the lending or borrowing of bank $i$ in the network $k ; g_{i, k}=\sum_{j=1}^{n_{k}} g_{i j, \kappa}$ is the number of direct links of $i ; \frac{1}{g_{i, k}} \sum_{j=1}^{n_{\kappa}} g_{i j, \kappa} q_{j, \kappa}$ is a spatial lag term; and $v_{i, k}$ is a random error term. The spatial lag term is equivalent to an autoregressive term in a time regression: a length-three connection in this model through lending connections is akin to a three period lag in a time series autoregressive model. This model is the so-called spatial lag model in the spatial econometric literature and can be estimated using the maximum likelihood (see, e.g. Anselin, 1988).

\section{Dynamic model}

In this section, we extend the model set out in Section 2 to include strategic link formation amongst banks. This step is crucial in that it permits us to include in our analysis not only the quantity and price choices amongst banks conditional on their existing network, but also their decisions on how to change the network structure itself. The model here will show the equilibrium outcome network structure conditional on these strategic choices. Such a model gives us the ability to validate that our static model results are reasonable insofar as they are not contradicted by strategic network formation incentives. It also allows us to investigate how strategic behavior can impact network structure and liquidity availability.

Our central modeling assumptions will be that links are formed based on the profitability tradeoff that emerges from the game in the static model. Effectively, banks know that the game will be played in the subsequent period and that all other banks are also making network formation decisions. Based on these, banks can choose whether or not to form a link; that is, to make a loan to a new bank. We will also specify an exogenous probability of link formation, $\alpha{ }^{9}$

To describe the network formation process we follow König et al. (2014a). Let time be measured at countable dates $t=1,2, \ldots$ and consider the network formation process $(G(t))_{t=0}^{\infty}$ with $G(t)=(N, L(t))$ comprising the set of banks $N=\{1, \ldots, n\}$ together with the set of links (i.e. loans) $L(t)$ at time $t$. We assume that initially, at time $t=1$, the network is empty. Then every bank $i \in N$ optimally chooses its quantity $q_{i} \in \mathbb{R}_{+}$as in the standard Cournot game with no network. Then, a bank $i \in N$ is chosen at random and with probability $\alpha \in[0,1]$ forms a link (i.e. loan) with bank $j$ that gives it the highest payoff. We obtain the network $G(1)$. Then every bank $i \in N$ optimally chooses its quantity $q_{i} \in \mathbb{R}_{+}$, and the solution is given by Equation (7). The profit of each bank is then given by Equation (8) and only

9 While we do not discuss in detail, this assumption can be relaxed in a number of ways. For example, König et al. (2010) show that a capacity constraint, what this model would interpret as a capital constraint, generates similar network patterns. 
depends on its Katz-Bonacich centrality, that is, its position in the network. At time $t=2$, again, a bank is chosen at random and with probability $\alpha$ decides with whom she wants to form a link while with probability $1-\alpha$ this bank has to delete a link if she has already one. Because of Equation (8), the chosen bank will form a link with the bank that has the highest Katz-Bonacich centrality in the network. And so forth.

As stated above, the randomly chosen bank does not create or delete a link randomly. On the contrary, it calculates all the possible network configurations and chooses to form (delete) a link with the bank that gives it the highest profit (reduces the least its profit). It turns out that connecting to the bank with the highest Katz-Bonacich centrality (deleting the link with the agent that has the lowest KatzBonacich centrality) is a best-response function for this bank. Indeed, at each period of time the Cournot game described in Section 2 is played and it rationalizes this behavior since the equilibrium profit is increasing in its Katz-Bonacich centrality (see Equation 8).

To summarize, the dynamics of network formation is as follows: At time $t$, a bank $i$ is chosen at random. With probability $\alpha$ bank $i$ creates a link to the most central bank while with complementary probability $1-\alpha$ bank $i$ removes a link to the least central bank in its neighborhood.

Characterization of equilibrium We would like to analyze this game and, in particular, to determine, in equilibrium, how many links banks will have. More importantly, we would like to describe the entire distribution of links for banks in the network. This degree distribution gives the percentage of banks with number of links (degree) $d=1, \ldots, n$. Recall that the decision to add or delete a connection to another bank is made based on bank optimization decisions that emerge from our static model.

Our results follow the work in König et al. (2014a) who show that, at every period, the emerging network is a nested split graph or a threshold network, whose matrix representation is stepwise. This means that agents can be rearranged by their degree rank and, conditional on degree $d \neq 0$, agents with degree $d$ are connected to all agents with degrees larger than $d$. Moreover, if two agents $i, j$ have degrees such that $d_{i}<d_{j}$, this implies that their neighborhoods satisfy $\mathscr{N}_{i} \subset \mathscr{N}_{j}$. Below, we will show how closely the theoretical patterns implied by this model are replicated in the data.

Denote by $N(d, t)$ the number of agents with degree $d \leqslant K / 2$ at time $t$. It can be shown that the dynamic evolution is given by:

$$
\begin{aligned}
& N\left(d, t^{\prime}+1\right)-N\left(d, t^{\prime}\right)=\left(\frac{1-\alpha}{n}\right) N\left(d+1, t^{\prime}\right)+\frac{\alpha}{n} N\left(d-1, t^{\prime}\right)-\frac{1}{n} N\left(d, t^{\prime}\right) \\
& N\left(0, t^{\prime}+1\right)-N(0, t)=\left(\frac{1-2 \alpha}{n}\right)-\frac{\alpha}{n} N(0, t)+\left(\frac{1-\alpha}{n}\right) N(1, t)
\end{aligned}
$$

These equations mean that the probability of adding connections to banks with degree $d$ is proportional to the number of nodes with degree $d-1$ (resp. $d+1$ ) when selected for node addition (deletion). The dynamics of the adjacency matrix (and from this the complete structure of the network) can be directly recovered from the solution of these equations. 
Since the complement $\bar{G}$ of a nested split graph $G$ is a nested split graph, we can derive the stationary distribution of networks for any value of $1 / 2<\alpha<1$ if we know the corresponding distribution for $1-\alpha$. With this symmetry in mind we restrict our analysis in the following to the case of $0<\alpha \leqslant 1 / 2$. Let $\{N(t)\}_{t=0}^{\infty}$ be the degree distribution with the $d$ th element $N_{d}(t)$, giving the number of nodes with degree $d$ in $G(t)$, in the $t$ th sequence $N(t)=\left\{N_{d}(t)\right\}_{d=0}^{n-1}$. Further, let $n_{d}(t)=N_{d}(t) / n$ denote the proportion of nodes with degree $d$ and let $n_{d}=\lim _{t \rightarrow \infty} \mathbb{E}\left(n_{d}(t)\right)$ be its asymptotic expected value (as given by $\mu$ ). In the following proposition (König et al., 2014a), we determine the asymptotic degree distribution of the nodes in the independent sets for $n$ sufficiently large.

\section{Proposition 4}

Let $0<\alpha \leqslant 1 / 2$. Then the asymptotic expected proportion $n_{d}$ of nodes in the independent sets with degrees, $d=0,1, \ldots, d^{*}$, for large $n$ is given by

$$
n_{d}=\frac{1-2 \alpha}{1-\alpha}\left(\frac{\alpha}{1-\alpha}\right)^{d}
$$

where

$$
d^{*}(n, \alpha)=\frac{\ln \left(\frac{(1-2 \alpha) n}{2(1-\alpha)}\right)}{\ln \left(\frac{1-\alpha}{\alpha}\right)}
$$

These equations precisely define the equilibrium degree distribution in the interbank market. The ability to reorganize - again based on incentives - allows one to understand how policy interventions may impact dynamic outcomes.

\section{Policy implications: which bank is key?}

In this section, we would like to use our static model of Section 2 to answer the following questions: when one bank is taken out of the network, or when one bank is hit by a shock, how do prices and quantities change? How does the network structure affect the answer? Which networks are more or less resilient? Can a policy maker improve resilience to shocks using some simple intervention. To address these questions, we will use the concept of "key player" introduced by Ballester et al. (2006). ${ }^{10}$ If a regulatory authority such as the ECB, the Federal Reserve Bank or the Banque de France needs to decide which bank should be bailed out because it is crucial for the "stability" of the bank system, then this bank will be the key player or key bank. In other words, the key bank is the one whose removal from the network would result in the largest reduction in total loan activity.

Formally, consider the previous model and denote by $Q^{*}(g)=\sum_{i=1}^{n} q_{i}^{*}$ the aggregate Nash equilibrium level of loans in network $g$, where $q_{i}^{*}$ is the Nash equilibrium loan quantity produced by bank $i$ and given by Equation (7). Denote also by $g^{[-i]}$ the network $g$ without bank $i$. Then, following Ballester et al. (2006), to determine the key bank, the planner will solve the following problem:

$$
\max \left\{Q^{*}(g)-Q^{*}\left(g^{[-i]}\right) \mid i=1, \ldots, n\right\}
$$

10 See Zenou (2015) for an overview on key players. 


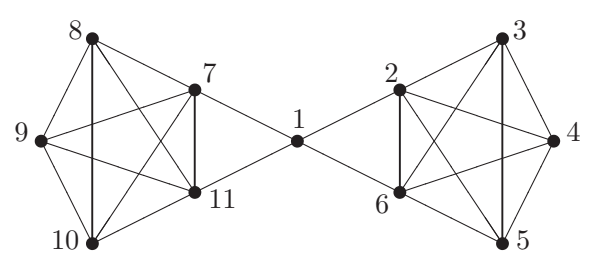

Fig. 3. A bridge network.

\begin{tabular}{lll}
\hline \hline Type & Banks \\
\hline 1 & 1 \\
2 & $2,6,7$, and 11 \\
3 & $3,4,5,8,9$, and 10 \\
\hline \hline
\end{tabular}

When the network $g$ is fixed, this is equivalent to:

$$
\min \left\{Q^{*}\left(g^{[-i]}\right) \mid i=1, \ldots, n\right\}
$$

Denote $\mathbf{M}(g, \phi)=(\mathbf{I}-\phi \mathbf{G})^{-1}$ so that the Katz-Bonacich centrality is defined as: $\mathbf{b}(g, \phi)=(\mathbf{I}-\phi \mathbf{G})^{-1} \mathbf{1}$. An element of $\mathbf{M}(g, \phi)$ corresponding to the cell $(i, j)$ is denoted by $m_{i j}$.

\section{Definition 1}

Assume $\phi \omega(g)<1$. The intercentrality or key-player centrality measure $\kappa_{i}(g, \phi)$ is defined as follows:

$$
\kappa_{i}(g, \phi)=\frac{\left[b_{i}(g, \phi)\right]^{2}}{m_{i i}}
$$

Ballester et al. (2006) derive the following result:

\section{Proposition 5}

A bank $i^{*}$ is the key player that solves (16) if and only if $i^{*}$ is the bank with the highest intercentrality in $g$, that is, $\kappa_{i^{*}}(g, \phi) \geqslant \kappa_{i}(g, \phi)$, for all $i=1, \ldots, n$.

The intercentrality measure, Equation (17), of bank $i$ is the sum of $i$ 's centrality measures in $g$, and $i$ 's contribution to the centrality measure of every other bank $j \neq i$ also in $g$. It accounts both for one's exposure to the rest of the group and for one's contribution to every other exposure.

To understand this concept, consider the network $g$ described in Figure 3 with eleven banks and three different types of players: 1, 2, and 3. This example was proposed by Ballester et al. (2006). For the sake of the exposition, we look at an undirected network so that if bank $i$ provides a loan to bank $j$ then the reverse is also true.

We distinguish three different types of equivalent actors in this network, which are the following: From a macro-structural perspective, type -1 and type -3 banks are identical: they all have four direct links (or loans), while type -2 banks have five direct links each. From a micro-structural perspective, however, bank 1 plays a critical role by bridging together two closed-knit (fully intraconnected) structures of five banks each. By removing bank 1, the network is maximally disrupted as these 
Table 1. Katz-Bonacich centralities versus intercentralities.

\begin{tabular}{clllllll}
\hline \hline \multirow{2}{*}{$\phi$} & \multicolumn{3}{c}{0.1} & & \multicolumn{3}{c}{0.2} \\
\cline { 2 - 4 } \cline { 5 - 7 } Bank type & $q_{i}$ & $b_{i}$ & $\kappa_{i}$ & & $x_{i}$ & $b_{i}$ & $\kappa_{i}$ \\
\hline 1 & 0.077 & 1.75 & 2.92 & & 0.072 & 8.33 & $41.67^{*}$ \\
2 & $0.082^{*}$ & $1.88^{*}$ & $3.28^{*}$ & & $0.079^{*}$ & $9.17^{*}$ & 40.33 \\
3 & 0.075 & 1.72 & 2.79 & & 0.067 & 7.78 & 32.67 \\
\hline \hline
\end{tabular}

two structures become totally disconnected, while by removing any of the type-2 banks, the resulting network has the lowest aggregate number of network links.

Let us determine the key bank in this network using Equation (17). If the choice of the key bank were solely governed by the direct effect of bank removal on aggregate loans, type-2 banks would be the natural candidates. Indeed, these are the ones that make the highest volumes of loans. But the choice of the key player needs also to take into account the indirect effect on aggregate loan quantities; that is, the reduction induced by the network restructuring that follows the removal of one bank from the original network. Because of its bridging role, bank 1 is also a possible candidate for the preferred policy target.

For banks of types 1,2, and 3, Table 1 computes the value of the volume of loans $q_{i}$, Katz-Bonacich centrality measures $b_{i}(g, \phi)$ and intercentrality measures $\kappa_{i}(g, \theta)$ for different values of $\phi$ and for $a=1$. In each column, a variable with a star identifies the highest value. ${ }^{11}$

First note that type-2 firms always display the highest Katz-Bonacich centrality measures. These banks have the highest number of direct connections. Besides, they are directly connected to the bridge bank 1, which gives them access to a very wide and diversified span of indirect connections. Altogether, they are the most $b$-central banks.

For low values of $\phi$, the direct effect on loan reduction prevails, and type-2 banks are the key players - those with highest intercentrality measure $\kappa_{i}$. In other words, they are the crucial banks in the system. When $\phi$ increases, however, the most active banks are no longer the key players. There indirect effects matter a lot, and removing bank 1 from the network results in the highest joint direct and indirect negative effect on aggregate loan volume. ${ }^{12}$

The individual Nash equilibrium loan quantities of the network game are proportional to the equilibrium Katz-Bonacich centrality network measures, while the key player is the bank with the highest intercentrality measure. As the previous example illustrates, these two measures need not coincide. This is not surprising, as both measures differ substantially in their foundation. Whereas the equilibriumKatz-Bonacich centrality index derives from strategic individual considerations, the intercentrality measure solves the planner's optimality collective concerns. In particular, the equilibrium Katz-Bonacich centrality measure fails to internalize all

11 We can compute the highest possible value for $\phi$ compatible with our definition of centrality measures, equal to $\widehat{\phi}=\frac{2}{3+\sqrt{41}} \simeq 0.213$.

12 Note that the network $g^{[-1]}$ has twenty different links, while $g^{[-2]}$ has nineteen links. In fact, when $\phi$ is small enough, the key-player problem minimizes the number of remaining links in a network, which explains why type -2 banks are the key player when $\phi=0.1$ in this example. 
the network payoff externalities banks exert on each other, while the intercentrality measure internalizes them all. More formally, the measure $\kappa(g, \phi)$ goes beyond the measure $\mathbf{b}(g, \phi)$ by keeping track of all the cross-contributions that arise between its coordinates $b_{1}(g, \phi), \ldots, b_{n}(g, \phi)$.

Definition 1 specifies a clear relationship between $d(g, \phi)$ and $\mathbf{b}(g, \phi)$. Holding $b_{i}(g, \phi)$ fixed, the intercentrality $d_{i}(g, \phi)$ of bank $i$ decreases with the proportion $m_{i i}(g, \phi) / b_{i}(g, \phi)$ of $i$ 's Katz-Bonacich centrality due to self-loops, and increases with the fraction of $i$ 's centrality amenable to out-walks.

It is important to understand the concept of key player in the interbank loan market. The key bank or key player is the one whose exit will be the most costly for the system in terms of total loan activity. However, the loss on total output (volume of loans) depends on the systemic risk parameter $\phi$, which measures the multiplier effects following the removal of a bank. ${ }^{13}$ In other words, if, in the network of Figure 3 , bank 1 would exit this market, then the loss of total activity in terms of loans will be the highest. This is true only if $\phi$ is high enough (here $\phi=0.2$ ) because, in that case, it will propagate beyond direct neighbors. To illustrate this point, consider again the network in Figure 3. It is easily verified that, when $\phi=0.1$, removing bank 1 leads to a decrease of $17.51 \%$ of total loans quantities while removing bank 2 leads to a decrease of $20.12 \%$. On the contrary, when $\phi=0.2$, these figures are $83.33 \%$ and $78.57 \%$, for bank 1 and 2 , respectively. ${ }^{14}$ In other words, when $\phi=0.2$, if bank 1 would exit from the interbank market, then the reduction in the total volume of loans in this network will be as high as $83.33 \%$. This is because, when bank 1 exits the market, it not only affects the banks it is directly linked with (banks 2, 6, 7, and 11) but also indirectly affects all the other banks in the interbank market. The way it affects the other banks is proportional to the "distance" in the network and it is discounted by a factor of $\phi^{k}$ for banks that are at a distance $k$ from bank 1 .

We can now easily answer the questions we asked at the beginning of this section. First, when a bank is taken out of the network, or when one bank is hit by a shock, we can see now how prices and quantities change and how it depends on the network structure. Indeed, using Equation (2), we can analyze the effect on prices when a bank exits the market since, in equilibrium, $\sum_{j \in N} q_{j}=a b(g, \phi) /[1+b(g, \phi)]$. In fact, it is easily verified that the difference in prices is inversely related to the difference in total quantities. For example, when $\phi=0.2$ and taking $\theta=1$, removing bank 1 or bank 2 from the market will lead to an increase of $81.82 \%$ and $77.11 \%$ in prices, respectively. It should also be clear that a policy maker can improve the resilience

13 In the peer-effect literature, $\phi$ is referred to as the social multiplier (see, in particular, Glaeser et al., 2003; Jackson \& Zenou, 2015; Liu et al., 2014).

14 Indeed, it easily verified that:

and

$$
\begin{aligned}
& \kappa_{1}(g, 0.1) \equiv b(g, 0.1)-b\left(g^{[-1]}, 0.1\right)=19.586-16.667=2.919 \\
& \kappa_{2}(g, 0.1) \equiv b(g, 0.1)-b\left(g^{[-2]}, 0.1\right)=19.586-16.305=3.281
\end{aligned}
$$

$$
\begin{gathered}
\kappa_{1}(g, 0.2) \equiv b(g, 0.2)-b\left(g^{[-1]}, 0.2\right)=91.667-50=41.667 \\
\kappa_{2}(g, 0.2) \equiv b(g, 0.2)-b\left(g^{[-2]}, 0.2\right)=91.667-51.334=40.333
\end{gathered}
$$


to shocks using a simple intervention based on the identification of the key bank. In a crisis setting, a policy maker would have additional rationale for support to a key institution. Or conversely, it would have a pre-crisis rationale for re-arranging the network importance to minimize the system's dependence on a given institution. Finally, network structure is also playing an important role since the key player will differ depending on the network structure. For example, in a star-shaped network, the key player will always be the central node, while, in other networks, like the one in Figure 3, it will depend on the magnitude of the systemic risk $\phi$. Indeed, to identify which networks are more or less resilient is a more complicated question that refers to a network design policy (Belhaj et al., 2014; König et al., 2014b). Given what we have shown here, it should be clear that regular networks (such as the complete network or the circle) should be more resilient since the exit of one bank from the market should have a relative lower impact on total activity since all banks have the same activity (and all have the same Katz-Bonacich centralities).

\section{Concluding remarks}

We have constructed two models of the interbank loan market, a static and dynamic one. Then, using these models, we have presented a measure of systemic risk in this market, which is a precise measure of the aggregate liquidity cost of a reduction in lending by an individual financial institution. This systemic risk measure is presented as an innovation vis-a-vis existing approaches. It is based on the foundation of a microfounded dynamic model of behavior. As well as, it provides a tool to understand the transmission of shocks that extends beyond default events and generalized price shocks. In the combination of these lies our tool; the competitive responses made by banks generate the transmission of shocks in our model and provide a tractable method of measuring and understanding systemic risk.

Our approach is designed to understand the role of network structure on interbank lending. As with any model, there are some limitations to the exercise. From a policy perspective, we emphasize the utility of using a structural approach to networks. To the extent that the model captures bank behavior, it allows policymakers the ability to test interventions with an eye both to how banks will optimize in the short-run and how networks will form and re-form under each assumption. We have also examined how a policymaker could monitor more closely the key bank, which is the bank that is crucial for the stability of the financial system.

An example is how one can interpret in our model with the imposition of the ECB's full allotment policy. This policy permitted banks to access credit lines from the ECB in unlimited quantities at a fixed rate. We can model this by removing many higher risk banks (or key banks) from the market. It is a straightforward result of the static model that the removal will lower average demand in this market as well as reduce average risk.

A second example is the use of exceptional capital cushions for SIFIs. Our approach allows one both to identify the SIFIs that are the largest contributors to systemic risk and determine what occurs if these banks face increased, and now binding, capital constraints. To identify the largest contributors, the static model indicates simply that the banks with the highest Katz-Bonacich centrality are those with the highest contribution. 
Using this information, an avenue for future research would be to evaluate optimal regulatory policy in the presence of networks. Given a particular objective function for the regulator, such as minimizing volatility or minimizing total systemic risk, the approach here could yield a set of capital constraints that solve the regulator's problem. Notice that these constraints would not necessarily have any of the cyclicality problems that a static, fixed constraint does. For example, the regulator could optimize over contribution to systemic risk over a period of time that includes recessions. Then, a capital cushion that depends on the contribution to risk and position in the network would vary against the cycle.

We note in closing that the core assumption of our paper that cost is decreasing the quantities of connections is central to our results. In other markets, and potentially in subsections of the market we discuss, this reverse could be true. The network implications would change if the cost function operated differently, but the insight that agent incentives are a function of the market structure would remain intact. We leave exploration of the details of this to future research.

\section{Acknowledgments}

We are very grateful to the Editors and two anonymous reports for helpful comments. We also thank Carefin - Bocconi Centre for Applied Research in Finance for financial support. Cohen-Cole also thanks the CIBER and the US Department of Education for support. Yves Zenou acknowledges financial support from the Riksbankens Jubileumsfond through research grant P13-0826:1. All errors are our own.

\section{References}

Acemoglu, D., Ozdaglar, A., \& Tahbaz-Salehi, A. (2015). Systemic risk and stability in financial networks. American Economic Review, forthcoming.

Adrian, T., \& Brunnermeier, M. (2009). CoVaR. Federal Reserve Bank of New York. Mimeo.

Albert, R., \& Barabási, A.-L. (2002). Statistical mechanics of complex networks. Review of Modern Physics, 74, 47-97.

Allen, F., Babus, A., \& Carletti, E. (2012). Asset commonality, debt maturity and systemic risk. Journal of Financial Economics, 104, 519-534.

Allen, F., \& Gale, D. (2000). Financial contagion. Journal of Political Economy, 108, 1-33.

Alves, I., Ferrari, S., Franchini, P., Heam, J., Jurca, P., Langfield, S., . . Vuillemey, G. (2013). The structure and resilience of the European interbank market. European Systemic Risk Board (ESRB) Occasional Paper No. 3.

Amini, H., Cont, R., \& Minca, A. (2012). Stress testing the resilience of financial networks. International Journal of Theoretical and Applied Finance, 15, 1250006.

Anselin, L. (1988). Spatial Econometrics: Methods and Models. Boston: Kluwer Academic Publishers.

Ballester, C., Calvo-Armengol, A., \& Zenou, Y. (2006). Who's who in networks. Wanted: The key player. Econometrica, 74, 1403-1417.

Barabási, A.-L., \& Albert, R. (1999). Emergence of scaling in random networks. Science, 286(5439), 509-512.

Belhaj, M., Bervoets, S., \& F. Deroïan (2014). Using network design as a public policy tool. Aix-Marseille School of Economics, Marseille, France. Unpublished manuscript. 
Bonacich, P. (1987). Power and centrality: A family of measures. American Journal of Sociology, 92, 1170-1182.

Boss, M., Elsinger, H., Summer, M., \& Thurner, S. (2004). Network topology of the interbank market. Quantitative Finance, 4(6), 677-684.

Boyson, N., Stahel, C., \& Stulz, R. (2010). Hedge fund contagion and liquidity shocks. Journal of Finance, 65, 1789-1816.

Cabrales, A., Gottardi, P., \& Vega-Redondo, F. (2013). Risk-sharing and contagion in networks. Mimeo.

Calvó-Armengol, A., Patacchini, E., \& Zenou, Y. (2009). Peer effects and social networks in education. Review of Economic Studies, 76, 1239-1267.

Cocco, J., Gomes, F., \& Martin, N. (2009). Lending relationships in the interbank market. Journal of Financial Intermediation, 18(1), 24-48

Cohen-Cole, E., Kirilenko, A., \& Patacchini, E. (2014). Trading network and liquidity provision. Journal of Financial Economics, 113, 235-251.

Craig, B., \& Von Peter, G. (2010). Interbank tiering and money center banks. FRB of Cleveland Working Paper No. 10-14.

Danielsson, J., Shin, H., \& Zigrand, J.-P. (2009). Risk appetite and endogenous risk. Mimeo.

Debreu, G., \& Herstein, I. N. (1953). Nonnegative square matrices. Econometrica, 21, 597-607.

Easley, D., \& Kleinberg, J. (2010). Networks, Crowds, and Markets: Reasoning About a Highly Connected World. Cambridge: Cambridge University Press.

Elliott, M. L., Golub, B., \& Jackson, M. O. (2014). Financial networks and contagion. American Economic Review, 104, 3115-3153.

Freixas, X., Parigi, B., \& Rochet, J. (2000). Systemic risk, interbank relations and liquidity provision by the central bank. Journal of Money, Credit and Banking, 32, 611-638.

Fricke, D., \& Lux, T. (2012). Core-periphery structure in the overnight money market: Evidence from the e-MID trading platform. Kiel Institute for the World Economy Working Paper No. 1759.

Furfine, C. (2003). Interbank exposures: Quantifying the risk of contagion. Journal of Money, Credit and Banking, 35(1), 111-128.

Gai, P., \& Kapadia, S. (2010). Contagion in financial networks. Bank of England Working Paper No. 383.

Glaeser, E. L., Sacerdote, B. I., \& Scheinkman, J. A. (2003). The social multiplier. Journal of the European Economic Association, 1, 345-353.

Goyal, S. (2007). Connections: An Introduction to the Economics of Networks. Princeton: Princeton University Press.

Iori, G., \& Jafarey, S. (2001). Criticality in a model of banking crisis. Physica A, 299, 205-212.

Iori, G., Jafarey, S., \& Padilla, F. (2006). Systemic risk on the interbank market. Journal of Economic Behavior and Organization, 61(4), 525-542.

Jackson, M. O. (2008). Social and economic networks. Princeton: Princeton University Press.

Jackson, M. O., \& Zenou, Y. (2015). Games on networks. In: P. Young, \& S. Zamir (Eds.), Handbook of Game Theory, volume 4 (pp. 91-157). Amsterdam: Elsevier Publisher.

Katz, L. (1953). A new status index derived from sociometric analysis. Psychometrika, 18, 39-43.

König, M., Tessone, C., \& Zenou, Y. (2010). From assortative to dissortative networks: The role of capacity constraints. Advances in Complex Systems, 13, 483-499.

König, M.D., Tessone, C., \& Y. Zenou (2014a). Nestedness in networks: A theoretical model and some applications. Theoretical Economics, 9, 695-752.

König, M.D., Liu, X., \& Y. Zenou (2014b). R\&D networks: Theory, empirics and policy implications. CEPR Discussion Paper No. 9872. 
Liu, X. Patacchini, E., Zenou, Y., \& Lee, L.-F. (2012). Criminal networks: Who is the key player? CEPR Discussion Paper No. 8772.

Liu, X., Patacchini, E., \& Zenou, Y. (2014). Endogenous peer effects: Local aggregate or local average? Journal of Economic Behavior and Organization, 103, 39-59.

Mistrulli, P. (2011). Assessing financial contagion in the interbank market: Maximum entropy versus observed interbank lending patterns. Journal of Banking and Finance, 35, 1114-1127.

Newman, M. E. J. (2010). Networks: An Introduction. Oxford: Oxford University Press.

Peltonen, T., Scheicher, M., \& Vuillemey, G. (2013). The network structure of the CDS market and its determinants. European Central Bank. Mimeo.

Pröpper, M., Lelyveld, I., \& Heijmans, R. (2008). Towards a network description of interbank payment flows. DNB Working Paper No. 177/May 2008, De Nederlandsche Bank.

Puhr, C., Seliger, R., \& Sigmund, M. (2012). Contagiousness and vulnerability in the Austrian interbank market. OeNB Financial Stability Report, No 24, Oesterreichische Nationalbank.

Soramäki, K., Bech, M., Arnold, J., Glass, R., \& Beyeler, W. (2007). The topology of interbank payment flows. Physica A, 379, 317-333.

Zenou, Y. (2015). Key players. In: Y. Bramoullé, B.W. Rogers \& A. Galeotti (Eds.), Oxford Handbook on the Economics of Networks, Oxford: Oxford University Press, forthcoming.

\section{Appendix: Theoretical results}

\section{Appendix A: The Katz-Bonacich network centrality}

The Katz-Bonacich network centrality measure is due to Katz (1953), and latter extended by Bonacich (1987). It provides a measure of direct and indirect links in a network. Effectively, a relationship between two banks is not made in isolation. If bank A lends money to bank B, and bank B already lends to bank $\mathrm{C}$, the strategic decisions of bank A will depend, in part on the strategic decisions of B. Of course, B's decisions will also be a function of C's. The Katz-Bonacich measure will help keep track of these connections and, as we will see in the subsequent section, has a natural interpretation in the Nash solution.

Let $\mathbf{G}^{k}$ be the $k$ th power of $\mathbf{G}$, with coefficients $g_{i j}^{[k]}$, where $k$ is some integer. The matrix $\mathbf{G}^{k}$ keeps track of the indirect connections in the network: $g_{i j}^{[k]} \geqslant 0$ measures the number of paths of length $k \geqslant 1$ in $g$ from $i$ to $j .{ }^{15}$ In particular, $\mathbf{G}^{0}=\mathbf{I}$, where $\mathbf{I}$ is the identity matrix. Denote by $\omega(\mathbf{G})$ the largest eigenvalue of $\mathbf{G}$.

\section{Definition 2}

Consider a network $g$ with adjacency $n$-square matrix $\mathbf{G}$ and a scalar $\phi \geqslant 0$ such that $\mathbf{M}(g, \phi)=(\mathbf{I}-\phi \mathbf{G})^{-1}$ is well-defined and non-negative. Let $\mathbf{1}$ be the $n$-dimensional vector of ones. Then, if $\phi \omega(\mathbf{G})<1$, the Katz-Bonacich centrality of parameter $\phi$ in $g$ is defined as:

$$
\mathbf{b}(g, \phi)=\sum_{k=0}^{+\infty} \phi^{k} \mathbf{G}^{k} \mathbf{1}=[\mathbf{I}-\phi \mathbf{G}]^{-1} \mathbf{1}
$$

15 A path of length $k$ from $i$ to $j$ is a sequence $\left\langle i_{0}, \ldots, i_{k}\right\rangle$ of players such that $i_{0}=i, i_{k}=j, i_{p} \neq i_{p+1}$, and $g_{i_{p} i_{p+1}}>0$, for all $0 \leqslant k \leqslant k-1$, that is, players $i_{p}$ and $i_{p+1}$ are directly linked in $\mathbf{g}$. In fact, $g_{i j}^{[k]}$ accounts for the total weight of all paths of length $k$ from $i$ to $j$. When the network is un-weighted, that is, $\mathbf{G}$ is a $(0,1)$-matrix, $g_{i j}^{[k]}$ is simply the number of paths of length $k$ from $i$ to $j$. 
These expressions are all well-defined for low enough values of $\phi$. It turns out that an exact strict upper bound for the scalar $\phi$ is given by the inverse of the largest eigenvalue of $\mathbf{G}$ (Debreu \& Herstein, 1953). The parameter $\phi$ is a decay factor that scales down the relative weight of longer paths. If $\mathbf{M}(g, \phi)$ is a nonnegative matrix, its coefficients $m_{i j}(g, \phi)=\sum_{k=0}^{+\infty \phi k} g_{i j}^{[k]}$ count the number of paths in $g$ starting from $i$ and ending at $j$, where paths of length $k$ are weighted by $\phi^{k}$. An element $i$ of the vector $\mathbf{b}(g, \phi)$ is denoted by $b_{i}(g, \phi)$. For all $\mathbf{b}(g, \phi) \in \mathbb{R}^{n}$, $b(g, \phi)=b_{1}(g, \phi)+\cdots+b_{n}(g, \phi)$ is the sum of its coordinates. Observe that, by definition, the Katz-Bonacich centrality of a given node is zero when the network is empty and is greater than 1 if the network is not empty. It is also null when $\phi=0$, and is increasing and convex with $\phi$.

\section{Appendix B: Nash equilibrium in loans}

Let us show how the first order condition can be written as a function of KatzBonacich centrality. For each bank $i=1, \ldots, n$, maximizing Equation (4) leads to:

$$
q_{i}^{*}=a-\sum_{j=1}^{n} q_{j}^{*}+\phi \sum_{j=1}^{n} g_{i j} q_{j}^{*}
$$

We can write this equation in matrix form to obtain:

$$
\mathbf{q}^{*}=a \mathbf{1}-\mathbf{J} \mathbf{q}^{*}+\phi \mathbf{G} \mathbf{q}^{*}
$$

where $\mathbf{J}$ is a $n \times n$ matrix of 1 . Since $\mathbf{J} \mathbf{q}^{*}=q^{*} \mathbf{1}$, this can be written as

$$
\begin{aligned}
\mathbf{q}^{*} & =(\mathbf{I}-\phi \mathbf{G})^{-1}\left(a-q^{*}\right) \mathbf{1} \\
& =\left(a-q^{*}\right) \mathbf{b}(g, \phi)
\end{aligned}
$$

Multiplying to the left by $\mathbf{1}^{t}$ and solving for $q^{*}$ gives:

$$
q^{*}=\frac{a b(g, \phi)}{1+b(g, \phi)}
$$

where $b(g, \phi)=\mathbf{1}^{t} \mathbf{b}(g, \phi)$. Plugging back $q^{*}$ into the previous equation gives

$$
\mathbf{q}^{*}=\frac{a}{1+b(g, \phi)} \mathbf{b}(g, \phi)
$$

which is Equation (7).

Existence and uniqueness of equilibrium are guaranteed by the condition $\phi \omega(\mathbf{G})<$ 1 , which ensures that the matrix $(\mathbf{I}-\phi \mathbf{G})^{-1}$ is non-singular. Interiority of equilibrium is straightforward to show.

\section{Appendix C: Network versus no network equilibrium}

Consider the same $n$ banks but without a network (i.e. $\phi=0$ ) so that there are no links (or loans) between them and $c_{i}=c_{0}$. In that case, the profit of each firm is given by:

$$
\pi_{i}=\theta q_{i}-\sum_{j=1}^{n} q_{i} q_{j}-c_{0} q_{i}
$$


The Nash equilibrium is such that:

$$
q_{i}^{*}=a-\sum_{j=1}^{n} q_{j}^{*}
$$

where $a \equiv \theta-c_{0}$. Summing the $n$ first-order conditions, we obtain:

$$
q^{N O *}=\left(\frac{n}{1+n}\right) a
$$

where $q^{*}=\sum_{j=1}^{n} q_{j}^{*}$, so that

$$
q_{i}^{N O *}=\frac{a}{1+n}
$$

Let us now define the Nash equilibrium quantities when banks are connected through a network. The first-order condition of Equation (6) is:

$$
q_{i}^{*}=a-\sum_{j=1}^{n} q_{j}^{*}+\phi \sum_{j=1}^{n} g_{i j} q_{j}^{*}
$$

or in matrix form

$$
\mathbf{q}^{*}=a \mathbf{1}-\mathbf{J q}^{*}+\phi \mathbf{G} \mathbf{q}^{*}
$$

where $\mathbf{J}$ is a $n \times n$ matrix of 1 . Since $\mathbf{J} \mathbf{q}^{*}=q^{*} \mathbf{1}$, this can be written as

$$
\mathbf{q}^{*}=a \mathbf{1}-q^{*} \mathbf{1}+\phi \mathbf{G} \mathbf{q}^{*}
$$

Multiplying to the left by $\mathbf{1}^{t}$, we get

$$
q^{*}=\left(\frac{n}{1+n}\right) a+\left(\frac{\phi}{1+n}\right) \mathbf{1}^{t} \mathbf{G} \mathbf{q}^{*}
$$

or equivalently:

$$
q^{N E T^{*}}=\left(\frac{n}{1+n}\right) a+\left(\frac{\phi}{1+n}\right) \sum_{i=1}^{n} \sum_{j=1}^{n} g_{i j} q_{j}
$$

By plugging back this equation into the first-order condition, we obtain:

$$
q_{i}^{N E T^{*}}=\frac{a}{(1+n)}+\phi \sum_{j=1}^{n} g_{i j} q_{j}^{*}-\left(\frac{\phi}{1+n}\right) \sum_{i=1}^{n} \sum_{j=1}^{n} g_{i j} q_{j}
$$

which is equivalent to:

$$
q_{i}^{*}=\frac{a}{(1+n)}+\left(\frac{n}{1+n}\right) \phi \sum_{j=1}^{n} g_{i j} q_{j}^{*}-\left(\frac{1}{1+n}\right) \phi \sum_{k \neq i}^{n} \sum_{j=1}^{n} g_{k j} q_{j}^{*}
$$

which is (9) and can be written as:

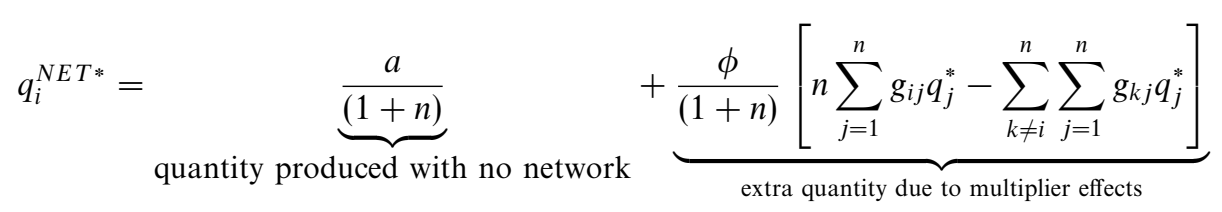

Let us now consider the dyad, i.e. the case of two banks $A$ and $B(n=2)$. Assume first that there is no network (i.e. $\phi=0$ ) so that no bank gives a loan to the other. 
In that case, using Equation (21), each bank will produce

$$
q^{N O *}=q_{A}^{N O *}=q_{B}^{N O *}=\frac{a}{3}
$$

Consider now the simplest possible network, that is each bank gives loans to the other bank, i.e. $g_{12}=g_{21}=1$. The adjacency matrix is ${ }^{16}$

$$
\mathbf{G}=\left(\begin{array}{ll}
0 & 1 \\
1 & 0
\end{array}\right)
$$

We easily obtain:

$$
\mathbf{b}(g, \phi)=\frac{1}{(1-\phi)}\left(\begin{array}{l}
1 \\
1
\end{array}\right)
$$

and thus the unique Nash equilibrium is given by:

$$
\mathbf{q}^{*}=\frac{a}{1+\frac{2}{1-\phi}} \mathbf{b}(g, \phi)=\frac{a}{3-\phi}\left(\begin{array}{l}
1 \\
1
\end{array}\right)
$$

that is

$$
q^{N E T^{*}}=q_{A}^{N E T^{*}}=q_{B}^{N E T *}=\frac{a}{3-\phi}
$$

Since $\phi<1$, then this solution is always positive and unique and

$$
q^{N E T *}=\frac{a}{3-\phi}>\frac{a}{3}=q^{N O *}
$$

In fact, we have:

$$
q^{N E T *}=q^{N O *}+\frac{a \phi}{3(3-\phi)}
$$

or equivalently

$$
q^{N E T *}=\frac{3}{(3-\phi)} q^{N O *}
$$

which is Equation (10).

16 There are two eigenvalues: $1,-1$ and thus $\omega(\mathbf{G})=1$. Thus the condition from Proposition $1, \phi \omega(\mathbf{G})<1$, is now given by: $\phi<1$. 